\title{
Discussion on the Relationship Types Between Lianmian Word and the Synonymous One-character Word
}

\author{
Juan $\mathrm{He}^{1,2, *}$ \\ ${ }^{1}$ College of Literature and Journalism, Sichuan University, Chengdu, Sichuan 610064, China \\ ${ }^{2}$ College of Humanities, Sichuan University of Science \& Engineering, Zigong, Sichuan 643000, China \\ *Corresponding author. Email: hejuanjk@126.com
}

\begin{abstract}
The phenomenon of Lianmian word sharing the similar sounds and identical meaning with a corresponding single word exists widely in Chinese, and the synonymous one-character word has the following types: the phonetic form of consonant clusters, the monosyllabic word, one word with double syllables, the syllable word, the morpheme of the word formation, etc. The most important relationship between Lianmian word and the corresponding monosyllabic word is that Lianmian word comes from the differentiation of consonant clusters, and the monosyllabic word comes from the differentiation of Lianmian word. That is, Lianmian words are the transitional stage of the development from consonant clusters to monosyllabic words.
\end{abstract}

\section{Keywords: Lianmian word, one-character word, consonant clusters, monosyllabic word}

\section{INTRODUCTION}

Lianmian word is an important language phenomenon in the history of Chinese vocabulary. Nowadays, Lianmian word is usually defined as a two-syllable monosyllabic words, which is composed of two Chinese characters, but cannot be explained separately. Its typical feature is that the two words only do not express meaning and cannot be used alone (or when used alone, it means something but has nothing to do with Lianmian word), such as "changyang" (徜徉), "chouchu" (踌躇), "paihuai" (徘徊), "choumou" (绸缪), "kunlun" (昆仑), etc. However, there is a wide range of Chinese such a kind of double-syllable words and monosyllabic words with similar pronunciation, that is, Lianmian word is related to the similar sound and meaning of a single syllable word, such as "hulun"(囷 图) "hun" (浑), "kulong" (窟察)-"kong" (孔). But in fact, there are the following situations between the words and their corresponding words:

- One character can be used alone, the meaning of which is the same as the Lianmian word, while another just means one syllable and can not be used alone (or when used alone, it has nothing to do with the meaning of the word), such as: "huyi" (狐 疑) -"yi" (疑), "minmian" (黾勉) -"mian" (勉), "guanpu" (莞蒲)一"pu" (蒲)，"jiaoliao" (椒聊) 一 "jiao" ( 椒), "jiuliu" ( 樛 流 ) - "jiu"( 樛 ), "mingling" (螟蛉) -"ming" (蚯), etc.

- The two characters of Lianmian word are just used separately in the corresponding context temporary, but not yet monosyllabic word, such as "huiwei" (荟蔚) - "hui" (荟), "wei" ( 蔚), for example, "Book of Songs· Cao Feng· Hou Ren" (《诗经・曹 风・候人》): "Huixi Weixi"(荟兮蔚兮); "tiaoda" (挑达)-Tiao(挑), Da(达), "Book of Songs· Zheng Feng· Zi Jin" (《诗经・郑风・子 衿》): "Tiaoxi Daxi (挑兮达兮)."

- Lianmian words have the same or similar pronunciation and meaning as singlecharacter words, that is, from the perspective of ancient sounds, the semantics of Lianmian word are the same as those of single words, and the sounds of single words are similar to those of the preceding character of Lianmian word, the rhymes and tones of single words are similar to those of the following character of Lianmian word, such as "jili" (薮㢣)-"ci" (茨), "zhong kui" (终 葵) -"zhui" (椎), "bolan" (孛缆) -"feng" (风), "dulou"(髑髅) - "tou" (头), "fuyao" (扶摇) "biao" (帣), etc. 
There is a common phenomenon in Chinese that the Lianmian word and the synonymous onecharacter word are clustered in sound and meaning.One Lianmian word contains two syllables with sound but no meaning, that means just the two syllables link together can become one soundmeaning word, which can not be separated to express a meaning [1]. But in fact, there are a large number of Lianmian words and the synonymous one-character word clustering phonetic and semantic in the ancient and modern Chinese, and even some words and the corresponding Lianmian syllable words are exactly the same. What is the relationship between the Lianmian word and the synonymous one-character word? Why does this happen?

As for the phenomenon that the Lianmian word and the synonymous one-character word cluster in phonetic and semantic, nowadays the common view is that one-character word are regarded as monosyllabic word, behind which the Lianmian words are often implied from monosyllabic words, which are produced after monosyllabic words. In fact these explanations are not proper, and there have been many studies showing that, there were consonant clusters in ancient Chinese, and Chinese was not originally developed from single syllable to multiple syllable[2]. Only Chinese characters are reserved for investigation nowadays. As the writing symbol of recording language, the development of Chinese characters lags far behind that of language. In addition, Chinese characters are ideographic characters, and the changes and differences in pronunciation are difficult to be directly expressed in form. According to our investigation, in the phenomenon of the phonetic and semantic clustering of Lianmian word and words, words are not always monosyllabic word, and the relationship with Lianmian word is not single.

\section{TYPES OF RELATIONSHIP BETWEEN LIANMIAN WORD AND THE CORRESPONDING WORD}

The relationship between Lianmian word and corresponding monosyllabic word has the following types:

\section{A. Some words in ancient Chinese are consonant clusters, and Lianmian words come from the differentiation of consonant clusters}

At present, the prevailing view is that ancient Chinese is dominated by monosyllabic words, and modern Chinese is dominated by disyllabic words, that is, the phonetic is based on monosyllabic consonants. Many studies have shown that there were complex consonants in ancient Chinese, but the complex consonants gradually disappeared later. "The differentiation of the initial consonants of the complex consonants in ancient Chinese probably began before the Chinese characters came into being, and the pre-Qin period continued long after the Chinese characters came into being".[3]In ancient Chinese, a Chinese character does not necessarily record a single consonant syllable, which may be the phonetic form of consonant clusters. Lianmian word comes from the differentiation of the corresponding consonants with consonant clusters, rather than from the monosyllabic words. On the contrary, Lianmian word will differentiate monosyllabic words. From the actual situation of the development of pronunciation, There were compound consonants in ancient Chinese, Lianmian word is the transition stage of the development from consonant clusters to monosyllabic word. At this stage, each syllable of Lianmian word has been a mono consonant and vowel double syllable structure, but has not yet been finally separated into a single consonant syllable. Then Lianmian word continues to differentiate, until differentiated into monosyllable words, as Wei Jiangong said: "The development of Lianmian word is the disappearance of polysyllable words in the polyphony relics, and the word become a voice with a character."[4]However, when Lianmian word is differentiated into monosyllabic word, even the original forms of Lianmian word are still used the same character. There are many these corresponding words in ancient Chinese, which reflect the development process of phonetics. For example, "孔"(klun)-"窟察

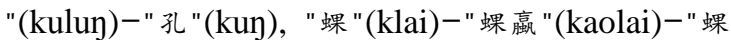
"(lai), "螳"(tluay)-"突郎"(tulan)-"螳"(tan), "团"(tluan) - " 突奕 "(tululan) - " 团 "(tuan), " 角 "(klok) - " 角落 "(koklak)-"角"(kok), etc. However, the polyphony has basically disappeared after the Eastern Han Dynasty, all evolved into monosyllabic words.

\section{B. Flexible use of Lianmian word}

The relationship between Lianmian word and monosyllabic words is not that monosyllabic words evolve into Lianmian word, on the contrary, Lianmian word decomposes monosyllabic words. However in ancient Chinese, the syllables of Lianmian word are temporarily used separately and separately in specific contexts to replace the meaning of the whole Lianmian word because of rhyme or the pursuit of textual changes, However, it is not a single syllable word, because after leaving the context, the single word has no real meaning (or has real meaning, but has nothing with the meaning of Lianmian word), which is divided into two situations:

- One character of the Lianmian word is also a monosyllabic word, which can replace whole Lianmian word 
"Book of Songs · ZhouSong · Zai Shan" (《诗 经 - 周颂 - 载茎》): "Youbiqixiang(有秚其香). ""bi"( 楿必)is"bibo"( 㿞䅗), means pleasant in fragrance.

"ChuCi·JiuSi" (《楚辞・九思》): "Wo peijiu xi zhonglu chu."(握佩㺵兮中路躇) "chu"(躇) is "chouchu" (踌躇), means hesitation.

- The two-syllable characters of Lianmian word are used separately in some context

Wang Yun of Qing Dynasty had already pointed out this kind of classification phenomenon in his works "Mao Shi Shuangsheng Dianyun Shuo" (《毛 诗双声叠韵说》). Because of the rhetoric requirement, the two-syllable characters of Lianmian word often appear separately in one sentence or one paragraph in the ancient literature, or even in a different chapter, and they called this kind of usage of Lianmian word as "variation" (变例), for example, "Book of Songs· Cao Feng· Hou Ren" (《诗经 - 曹 风・候人》): "Huixi Weixi (荟兮蔚兮), Nanshan Zhaoji (南山朝济)." "Huiwei" (荟蔚) was originally a whole word, but here used separately, doesn't mean it's a two-syllable word "hui" (荟) and "wei" (蔚).

Although Lianmian word cannot be regarded as a combination of two monosyllabic words, in ancient Chinese poetry and prose, temporary singleuse and separate-use phenomena often appear in concrete application due to the rhetorical requirements of repetition, phonological harmony and sentence pattern symmetry. But the separate characters did not yet become a word, because they can not be established after leaving the corresponding context. That is to say, the character still retained the whole Lianmian word, but that doesn't mean Lianmian word is a two-word combination. To some extent, this kind of variant usage of Lianmian word in ancient Chinese provides the conditions for further differentiation and falling off.

\section{The syllable Morphemization of Lianmian word}

At the beginning of the differentiation of Lianmian word, some were frequently used separately or flexibly, which made the syllables in the Lianmian word became morphemation and gave rise to the words related to the morphemic syllables, the syllables, which had no meaning became a syllable that had meaning, and still could not be used alone but just only be used as morphemes, for example, "hudie" (蝴蝶) -"die" (蝶), "zhizhu" (蜘蛛) "zhu" (蛛), "chanchu" (蟾蜍)-"chan" (蟾), "qilin" (麒麟) - "lin"(麟) and so on, which is now the most important form of monosyllabic words.

\section{Single syllable word notes Lianmian word entry}

In dictionaries, a character is often used as a prefix to identify the whole Lianmian word, for example,"Shuowen · Chongbu" (《说文・虫部》): "Bian(蝙), bianfu ye(蝙蝠也)." And "Fu(蝙), bianfu(蝙蝠也)." But the single character is not monosyllabic, but only the phonetic characters which do not express meaning. Actually these phonetic characters are often used as the head of words in dictionaries to mark the entries of Lianmian word. It only shows the phonetic relationship with disyllabic words, the subsequent interpretation is of the whole word made up of phonetic characters, rather than single-syllable words.

\section{E. Chinese character may be disyllable}

In ancient Chinese dictionaries, there is another kind of phenomenon in the enumeration of Lianmian word, in which only one of the two characters appears in the Lianmian word, but not the other (or appears another character, but the meaning has nothing to do with the Lianmian word), the reason for this is that at the beginning of language differentiation, phonetics became monosyllabic words, but the form did not keep up with the rhythm, for example,"Shuowen · Chongbu" (《说文 - 虫部》): "Shuai (幦), xishuai ye(悉璧也)." And he pointed out that in this dictionary, there is only "shuai" (晿), but no"xi" (悉), because the "shuai" (䖵) has both "xi" (悉) and "shuai" (䖵) sounds. [5]

\section{F. Lianmian word differentiates into monosyllabic word}

Disyllabic words differentiated into monosyllabic words when the pattern of Chinese characters as monosyllabic syllables became stable, the original consonant consonants had been differentiated and became disyllabic words of two characters. Disyllabic words continued to develop and evolved into monosyllabic words, some of them are directly falling off a syllable word, and another syllable word has evolved into a single syllable word, such as: "minmian" (黾勉) "mian"（勉）, "jiaoliao"( 椒聊）－ "jiao"（椒）, "mingling" (螟蛉) - "ming" (蚯), "tuanluan" (团栾) "tuan"(团), etc. Another way is combining sounds of Lianmian word to produce new monosyllabic word, such as "bulü" (不律)-"bi" (笔), "fuyao" (扶摇)

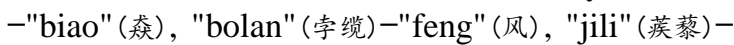
"ci" (茨), "dulou" (鹘髅) -"tou" (头) and so on. At this phase, each syllable is already a single consonant syllable. The ancient initial consonants of the monosyllabic word are the same (or similar) as that of the first syllable of a Lianmian word, and the ancient rhyme and tone is the same (or similar) as that of the second syllable of a Lianmian word. 
Most of these Lianmian word have the relationship of reiterative (or disyllabic). And the relationship between Lianmian word and the corresponding monosyllabic words is not that the monosyllabic words constitute the Lianmian word, on the contrary, the monosyllabic words are differentiated from the Lianmian word.

Monosyllabic words are differentiated from the Lianmian word, while in writing the monosyllabic words often appear earlier than Lianmian word. This paper holds that the sequence of writings does not fully represent the sequence of language production. Firstly, in the phenomenon of the sound and meaning correspondence between Lianmian word and monosyllabic word, the monosyllabic word may consonant clusters sound form, so it will appear earlier than the Lianmian word, later evolved into a monosyllabic word, but still used the original character. Secondly, the written record lags far behind the development of language. As the Lianmian word is original underlying language, and most of them have sounds but no writings. They are occasionally recorded in the writings and often change in sound and character. Monosyllabic words are mostly written language and their sounds and characters are more stable. And in ancient Chinese, monosyllabic words are dominant, so the written records are preserved earlier and more completely than Lianmian word.

\section{CONClusion}

In ancient and modern Chinese, there is a widespread phenomenon of homonymy between Lianmian word and single-character word, but because Chinese characters do not represent phonetic characters, the initial phonetic situation cannot be directly reflected from the character form. According to our investigation, in the phenomenon of synonyms between Lianmian word and singlecharacter word in ancient times, the corresponding word may be the phonetic form of consonant clusters, the monosyllabic word, one word with double syllables, the syllable word, the morpheme of the word formation, etc. Especially the relationship between the two kinds of words, Lianmian word comes from the differentiation of the corresponding consonants with consonant clusters, rather than from the monosyllabic words, On the contrary, Lianmian word will differentiate monosyllabic words. Merely the single-syllable words in modern Chinese are already disyllabic words., there is only a trace of homonymy between Lianmian word and single-character word nowadays. But it does not mean that all the Lianmian words have been differentiated, and there are still many concatenated words that have not yet been matched by single syllables, such as

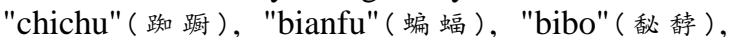
"chanchu" (蟾蜍)，"dianxi"(殿屎)，"luli"( 陆离), "qiaocui" (憔悴) and so on, and they may continue to differentiate in the future.

\section{References}

[1] Zhou Bingjun. Outline of ancient ChineseM.Changsha: Hunan Education Press, 1998:236.

[2] Chen Duxiu. The Compilation of BinomenA// Chen Duxiu's essays on phonology. Beijing: Zhonghua Book Company,2001:113.

[3] Shang Yuhe. The existence of "Feng Yue BoLan" (风曰孛 缆) and the initial consonants of ancient Chinese compound consonants A//Linguistics Series(the eighth series). Beijing: The Commercial Press, 1981:77.

[4] Wei Jiangong. A Study on the Ancient Sound System.Beijing: Zhonghua Book Company, 1996:155.

[5] Zhang Taiyan. Guogu Lunheng. Beijing: The Commercial Press, 2010:38.

[6] Xu, Shen \& Yucai Duan: Shuo Wen Jie Zi Zhu(Photocopy Book). Shanghai: Shanghai Ancient Books Publishing House.1981. 